\title{
Cardiomyopathy and autonomic neuropathy in hereditary amyloidosis: Defining the cart and the horse
}

\author{
Prem Soman, MD, PhD, MASNC \\ a Division of Cardiology and The Heart and Vascular Institute, University of Pittsburgh Medical \\ Center, Pittsburgh, PA
}

Received Nov 29, 2018; accepted Nov 29, 2018

doi: $10.1007 / \mathrm{s} 12350-018-01560-4$

\section{See related article, pp. 1774-1784}

Autonomic dysfunction is common in patients with AL amyloidosis and hereditary (mutant) ATTR polyneuropathy and cardiomyopathy (m ATTR). It has also been infrequently described in wild-type ATTR cardiomyopathy. ${ }^{1,2}$

As described by Wang et $\mathrm{al}^{3}{ }^{3}$ the term autonomic dysfunction implies the presence of one of more of an exhaustive list of symptoms including blurred vision or glare (pupillary dysfunction), dry eyes, or mouth (impaired cholinergic sympathetic innervation to lacrimal or salivary glands), gustatory sweating or heat intolerance (abnormal sudomotor function), cold hands and feet (peripheral sympathetic dysfunction), orthostatic hypotension, gastrointestinal symptoms of early satiety, alternating diarrhea and constipation, urinary frequency, urgency retention or incontinence, and sexual dysfunction. In their study of autonomic dysfunction in 65 patients with biopsy-proven amyloidosis, ${ }^{3}$ Wang et al used an autonomic reflex screen (ARS), a group of tests designed to test the adrenergic (blood pressure and heart-rate response to tilt, beat-to-beat blood pressure response to the Valsalva maneuver), cardiovagal (heartrate response to deep breathing at 6 breaths/min, the Valsalva ratio calculated from the maximal and minimal heart rates during the Valsalva maneuver), and sudomotor (the quantitative sudomotor axon reflex test,

Reprint requests: Prem Soman, MD, PhD, MASNC, Division of Cardiology and The Heart and Vascular Institute, University of Pittsburgh Medical Center, A-429 Scaife Hall, 200 Lothrop Street, Pittsburgh, PA 15213; premsoman@usa.net

J Nucl Cardiol 2020;27:1785-6.

$1071-3581 / \$ 34.00$

Copyright (c) 2018 American Society of Nuclear Cardiology.
QSART) components of autonomic dysfunction. The results of these individual components were integrated into the composite autonomic severity score (CASS), resulting in a semiquantitative measure of autonomic dysfunction (mild, moderate, and severe-depending on the score). Most other studies of autonomic dysfunction in amyloidosis patients have used a more limited symptom screen and battery of tests. While Wang et al describe comprehensive autonomic testing, their study also highlights a major difficulty with the assessment of autonomic function, namely the variety and nonspecific nature of potential symptoms, and the range of testing needed for a complete evaluation of all subcomponents.

I-123 mIBG imaging has been used to evaluate myocardial sympathetic function more objectively. ${ }^{4}$ The tracer was approved by the US FDA for this purpose in 2013. The uptake of I-123 mIBG by the myocardium is a reflection of the integrity of the NET-1 uptake mechanism, which is impaired in patients with heart failure and presumably, in other causes of sympathetic nerve terminal dysfunction or denervation. Its retention by the myocardium is impaired under conditions of an elevated sympathetic tone. These kinetic parameters are assessed by early (15 $\mathrm{min}$ ) and late (4 hours) heart-to-mediastinal $\mathrm{H} / \mathrm{M})$ ratios, and a washout rate. Assessment of regionality of dysfunction is performed by SPECT imaging. ${ }^{4}$

The pathogenesis of autonomic neuropathy in amyloidosis is attributed to infiltration by amyloid fibrils. While this is a logical hypothesis, it is a difficult one to prove. Global abnormalities of I-123 mIBG uptake (HM and washout ratios) may simply reflect heart failure, and regional abnormalities demonstrated thus far have been nonspecific. Histopathological evidence of nerve and ganglionic plexus infiltration has been demonstrated in the GI tract of patients with autonomic symptoms and amyloidosis, ${ }^{5}$ but other mechanisms such as neuronal ischemia due to vessel wall infiltration by 
amyloid and toxic effect of pre-fibrillar TTR oligomers have also been postulated. ${ }^{6,7}$

It is in this context that Jonker and colleagues ${ }^{8}$ report on a study of clinical findings, cardiac biomarker assessment, and I-123 mIBG findings in patients with histologically proven mATTR amyloidosis and asymptomatic carriers of a TTR mutation $(48 \% \mathrm{~V} 30 \mathrm{M})$. The stated objective of this study was to determine whether abnormal mIBG findings in these patients and carriers are an indication of autonomic dysfunction specifically or simply an evidence of cardiac involvement in amyloidosis. To explore this objective, the investigators retrospectively evaluated data from 26 mATTR patients and 13 carriers. Some critical definitions should be noted. Cardiomyopathy was defined as NT-proBNP $>365 \mathrm{ng} / \mathrm{l}$ in the presence of normal kidney function, with no consideration of echo or Tc-99 m HDP bone scintigraphy findings. Autonomic neuropathy was defined by a variety of cardiovascular, gastrointestinal, and urogenital symptoms, a battery of bedside maneuvers, and a test for sympathetic skin response. The 29 subjects were then classified into groups (necessarily small) based on the presence or the absence of cardiomyopathy and autonomic neuropathy, and the results of the Tc-99 m HDP (abnormal defined as any cardiac uptake) and I-123 mIBG scans (abnormal defined as late HM ratio $<2.0$ or washout rate $>20 \%$ ). Cardiac troponin T levels were measured, but not used to classify patients.

Summary findings were that:

1. Of the 39 enrolled subjects (patients and carriers), 9 had cardiomyopathy, 13 autonomic neuropathy, 16 cardiac Tc-99 m HDP uptake and 18, abnormal I-123 mIBG.

2. Patients with "cardiomyopathy" (BNP $>365 \mathrm{ng} / \mathrm{l})$ had lower HM ratio irrespective of the presence of autonomic neuropathy.

3. The late HM and washout ratios inversely correlated with NT-proBNP and cardiac Tc-99 m HDP uptake.

The authors conclude that abnormal I-123 mIBG uptake by the myocardium in patients with histologyproven mATTR amyloidosis or carriers of a mutant TTR gene is indicative of cardiomyopathy rather than specifically, autonomic neuropathy. This conclusion is presumably based on the relative prevalence of cardiomyopathy, autonomic dysfunction, and abnormal I123 mIBG results, as defined by the authors for the purposes of this study. And therein lies the major difficulty with the authors' conclusions.

The definition of amyloid cardiomyopathy based on a NT-pro BNP value alone, without consideration of echo, ECG, and bone scintigraphy results is restrictive and unconventional. The patients, who had Tc-99 m
HDP uptake or typical ECG or echo abnormalities with histological evidence of amyloidosis, should surely be diagnosed with cardiomyopathy even in the absence of biomarker elevation. What was demonstrated in this study is that in subjects with an elevated NT-pro BNP, global abnormalities in HM and washout ratio may occur in the absence of evidence of autonomic neuropathy. That these abnormalities reflect the neurohormonal perturbations of heart failure, and not specifically autonomic dysfunction, is consistent with our understanding of mIBG kinetics.

Therefore, can we draw any conclusions from this small study? The validity of the conclusion that I-123 mIBG abnormalities occur early in amyloidosis and thus, may have diagnostic utility for cardiomyopathy is inherently dependent on the definition of cardiomyopathy and therefore a self-fulfilling prophecy for this study. Do I-123 mIBG abnormalities occur before other manifestations of cardiomyopathy, or do these abnormalities simply reflect the altered neurohormonal milieu of heart failure? In other words, which is the cart and which, the horse? In this case, the answer depends on how the cart and horse are defined!

\section{Disclosure}

Dr. Prem Soman has received fees for serving on the Advisory Board of Alnylam Pharma.

\section{References}

1. Slart R, Glaudemans A, Hazenberg BPC, Noordzij W. Imaging cardiac innervation in amyloidosis. J Nuclear Cardiol. 2017. https://doi.org/10.1007/s12350-017-1059-9.

2. Pinney JH, Whelan CJ, Petrie A, et al. Senile systemic amyloidosis: clinical features at presentation and outcome. J Am Heart Assoc. 2013;2:e00098.

3. Wang AK, Fealey RD, Gehrking TL, Low PA. Patterns of neuropathy and autonomic failure in patients with amyloidosis. Mayo Clin Proc. 2008;83:1226-30.

4. Soman P, Travin MI, Gerson M, Cullom SJ, Thompson R. I-123 MIBG cardiac imaging. J Nuclear Cardiol. 2015;22:677-85.

5. Anan I, El-salhy M, Ando Y, Terazaki H, Suhr OB. Comparison of amyloid deposits and infiltration of enteric nervous system in the upper with those in the lower gastrointestinal tract in patients with familial amyloidotic polyneuropathy. Acta Neuropathol. 2001; 102:227-32.

6. Sousa MM, Cardoso I, Fernandes R, Guimaraes A, Saraiva MJ. Deposition of transthyretin in early stages of familial amyloidotic polyneuropathy: evidence for toxicity of nonfibrillar aggregates. Am J Pathol. 2001;159:1993-2000.

7. Goldstein DS. Cardiac dysautonomia and survival in hereditary transthyretin amyloidosis. JACC Cardiovasc Imaging. 2016;9:1442-5.

8. Jonker DL, Hazenberg PCB, Nienhuis HLA, et al. Imaging cardiac innervation in hereditary transthyretin (ATTRm) amyloidosis: a marker for neuropathy or cardiomyopathy in case of heart failure? $\mathrm{J}$ Nuclear Cardiol. 2018. https://doi.org/10.1007/s12350-018-01477-y. 